\title{
Stochastic Analysis of Reliability Indices for a Redundant System under Poisson Shocks
}

\author{
Mohamed S. El-Sherbeny \\ Department of Mathematics, \\ Faculty of Science, \\ Helwan University, Egypt
}

\author{
Elsayed Elshoubary \\ Department of Basic Science, \\ Pyramid Higher Institute for Engineering and \\ Technology, Egypt
}

\begin{abstract}
This paper studies the effectiveness of repairman on system consisting of two operating repairable units. The system fail due to external factor like Poisson shocks that occur in different times. The arrivals of the shocks follow a Poisson process and the magnitude of a shock is an independent random variable following a known distribution. Repair time, the length of repairman's vacation and recall time are arbitrary distributions. Certain important results have been derived: the reliability, mean time to failure, steady-state availability and steady-state frequency of the system using supplementary variable technique. Special case is derived from the system.
\end{abstract}

\section{Keywords}

Poisson shock, Steady-state availability, Steady-state frequency, Mean time to failure, Supplementary variable technique.

\section{INTRODUCTION}

A Poisson process is the simplest stochastic process that arises in many applications for arrival processes. It is of great importance to know the properties of a Poisson process and to learn how to apply the process to reliability models. Idea of shock model has been studied in the last decades. For example, Li et al. [1] deal with the study of complex systems consisting of $n$ i.i.d. units with a $\delta$-shock model. M. Salah ELSherbeny [2] studied stochastic analysis of a two non-identical unit parallel system with different types of failures subject to preventive maintenance and repairs. Cost (benefit) models in the machine repair problem have been investigated by several authors including M. Salah EL-Sherbeny et al. [3]. Mahmoud and Moshrefa [4] studied the stochastic analysis of a twocomponent cold standby system considering hardware failure, human error failure and preventive maintenance. Optimal system for series systems have been proposed, for example, optimal system for series systems with mixed standby components by M. Salah EL-Sherbeny [5, 6]. Doshi [7] studied a comprehensive survey on vacation system models. Ke and Wang [8] investigated a machine repair problem with two vacation policies (single vacation and multiple vacations), both of which were based on a queuing theory viewpoint. Goel \& Shrivastava [9] studied comparison reliability characteristics of two systems with bivariate exponential lifetimes. Jia and $\mathrm{Wu}[10]$ developed a replacement policy for a repairable system with its repairman taking multiple vacations. Yuan and $\mathrm{Xu}$ [11] provided a repairable system with a repairman, who can take multiple vacations. Wu and $\mathrm{Wu}$ [12] investigated a two-component cold standby repairable system with its repairman taking single vacation and the system might be attached by cumulative shocks. Yutian Chen et al [13] studied two-component cold standby system, in which the operating component may fail due to the intrinsic factors or external factors; besides, the repairman can take vacation. Systems studied are useful for engineers and interested in maintenance in construction models to avoid unnecessary expenses and reduction of safety standards.

This paper interested in studying the effect of the repairman on repairable system consisting of two operating units. Special case is derived and makes comparison with the main system. In the main system the repairman has a choice between go vacation or stay in initial state of this system and when he is on vacation we must recall him in case of the presence of the failure. In special case system the repairman already on vacation in initial state and we must recall him when the failure occurs. The contributions of this paper are twofold. The first is to derive some measures of system effectiveness like the reliability, Mean time to failure, the steady-state failure frequency of the system and the steadystate availability. The second is to study the performance of the repairman on systems is shown graphically.

\section{ASSUMPTIONS}

The following assumptions are associated with the system

1. The system consists of two operating non-identical parallel units.

2. The system remains operating even if a single unit operates.

3. The system suffers from an external factor like Poisson shocks that occur in different times.

4. The distributions of vacation length of the repairman, recall time of repairman and repair time are arbitrary while the failure time follows the exponential distribution.

5. In the initial state the repairman has a choice between go on vacation or stay idle until the emergence of the first failure.

6. We must recall the repairman from vacation when the failure occurs for any unit.

7. The failure unit will be repaired immediately with the presence of the repairman.

8. The repair rule is first- come- first- service.

9. The arrivals of the shocks follow a Poisson process $\{\mathrm{N}(\mathrm{t}), \mathrm{t} \geq 0\}$ with the intensity $\lambda_{\mathrm{i}}>0$.

10. The quantity of each shock, $\hat{X}$, is an independent random variable with distribution function $F$.

11. The shock affects the units with manly the unit will fail when values of the shocks exceeds a threshold.

12. The threshold of unit $i$ is a non-negative random 


$$
\begin{aligned}
& \text { variable } \tau_{i} \text { with a distribution function } \\
& \psi_{i},(\mathrm{i}=1,2)
\end{aligned}
$$

13. Suppose $Y_{i}(i=1,2)$ as unit $i$ is repair time, $Z$ as the vacation length of the repairman and $K$ as the recall time of the repairman. their distributions are:

$$
\begin{aligned}
& H_{i}(t)=\int_{0}^{t} h_{i}(x) d x=1-\ell^{-\int_{0}^{t} \mu_{i}(x) d x}, \\
& \mathrm{E}\left(\mathrm{Y}_{i}\right)=\frac{1}{\mu_{i}}, \quad \bar{H}_{i}(t)=1-H_{i}(t) \quad(\mathrm{i}=1,2),
\end{aligned}
$$$$
V_{1}(t)=\int_{0}^{t} v_{1}(x) d x=1-\ell^{-\int_{0}^{t} \varphi(x) d x}
$$$$
\mathrm{E}(\mathrm{Z})=\frac{1}{\varphi}, \quad \bar{V}_{1}(t)=1-V_{1}(t)
$$$$
V_{2}(t)=\int_{0}^{t} v_{2}(x) d x=1-\ell^{-\int_{0}^{t} \alpha(x) d x}
$$$$
\mathrm{E}(\mathrm{K})=\frac{1}{\alpha}, \quad \bar{V}_{2}(t)=1-V_{2}(t)
$$

respectively.

14. The system is down if the two units fail,

15. After the repair, the unit is as good as new.

\section{NOTATION AND STATES OF THE SYSTEM}

\subsection{Notations}

$\begin{array}{ll}X_{1}(t) & \begin{array}{l}\text { Random variable denoting the elapse } \\ \text { vacation time when the } \\ \text { repairman is taking a vacation at tim } t \\ X_{2}(t)\end{array} \\ Y_{i}(t) & \begin{array}{l}\text { Random variable denoting the recalli } \\ \text { time from vacation at time } t\end{array} \\ & \text { Random variable denoting the elapse } \\ h(t), H(t) & \text { repair time at time } t, \forall i=1,2 . \\ v_{1}(t), \mathrm{V}_{1}(t) & \begin{array}{l}\text { p.d.f. and c.d.f. of the repair time } \\ \text { of a repairman }\end{array} \\ v_{2}(t), \mathrm{V}_{2}(t) & \begin{array}{l}\text { p.d.f. and c.d.f. of the recall time } \\ \text { of a repairman }\end{array} \\ f(t), F(t) & \begin{array}{l}\text { p.d.f. and c.d.f. of the magnitude } \\ \text { of each shock }\end{array} \\ P_{i}(t, x) & \begin{array}{l}\text { p.d.f. and c.d.f. of the system is in } \\ \end{array}\end{array}$

$Q_{i}(t, x)$

$P_{i}(t, y)$

$Q_{i}(t, y)$

$P_{0}(t, u) \quad Q_{0}(t, u) \quad$ p.d.f. and c.d.f. of the system is in state

$S_{0}$ at period $t$ and has an elapsed vacation

time of $u$

$P_{i}(t, z)$

$Q_{i}(t, z)$

$\varphi(u)$

$\mu_{1}(x)$

$\mu_{2}(y)$

$\alpha(z)$

$\lambda_{i}$

$r_{i}$

p.d.f. and c.d.f. of the system is in state

$i=5,6,7,10$ at period $t$ and has recalling

time of $z$

Vacation time distribution function

The repair rate of unit $\mathrm{A}$

The repair rate of unit $\mathrm{B}$

Recall time distribution function

Intensity of shock on unit $i \forall i=1,2$.

The probability that one shock

causes unit $i$ to fail is:

$$
\begin{aligned}
& r_{i}=P\left(\widehat{X}>\tau_{i}\right)=\int_{0}^{\infty} P\left(\tau_{i}<\widehat{X} / \widehat{X}\right. \\
& =\widehat{x}) d P(\widehat{X} \leq \widehat{x})=\int_{0}^{\infty} \psi_{i}(\widehat{x}) d F(\widehat{x}), \\
& (i=1,2)
\end{aligned}
$$

$$
\begin{array}{ll}
h^{*}(s) & \text { Laplace transform of } h(t) \\
p_{i}(t) & \text { Probability that the system is } \\
& \text { in state } i \text { at time } t
\end{array}
$$

\subsection{Symbols for the states of the system}

These symbols are common for two systems.

$A_{N} \quad$ Component $A$ in normal and operative mode.

$B_{N} \quad$ Component $B$ in normal and operative mode.

A. Component $A$ in failure mode due to shock failure and under repair.

$B_{r} \quad$ Component $B$ in failure mode due to shock failure and under repair.

$A_{R} \quad$ Component $A$ in repair of failed unit is continued from earlier state.

$B_{R} \quad$ Component $B$ in repair of failed unit is continued from earlier state.

$A_{w r} \quad$ Component $A$ in failed unit waiting for the repair. 
$B_{w r} \quad$ Component $B$ in failed unit waiting for the repair.

$A_{W R} \quad$ Component $A$ in waiting for the repair unit is continued from earlier state.

$B_{W R} \quad$ Component $B$ in waiting for the repair unit is continued from earlier state.

Considering these symbols, the system can be in any of the following states which are the description of system

Up states: $S_{0}=\left(A_{N}, B_{N}\right), \quad S_{1}=\left(A_{N}, B_{r}\right)$, $S_{2}=\left(A_{r}, B_{N}\right), S_{5}=\left(A_{N}, B_{w r}\right), S_{6}=\left(A_{w r}, B_{N}\right)$

Idle state: $S_{3}=\left(A_{N}, B_{N}\right)$

Down $\quad$ states: $\quad S_{4}=\left(A_{R}, B_{w r}\right), \quad S_{7}=\left(A_{W R}, B_{w r}\right)$, $S_{8}=\left(A_{w r}, B_{R}\right)$, $S_{9}=\left(A_{r}, B_{W R}\right)$,

$S_{10}=\left(A_{w r}, B_{W R}\right), S_{11}=\left(A_{W R}, B_{r}\right)$

Figure 1 describes the state transition of the system according to a Markov chain, where the states $0,1,2,5$ and 6 are working states; while the states $4,7, \ldots, 11$ are failure states and the state 3 is idle state.

\section{CALCULATIONS OF SYSTEM}

\subsection{The steady-state availability}

This section investigates availability behavior of the system. Using supplementary variable technique

$$
\begin{aligned}
& \left(\frac{\partial}{\partial t}+\frac{\partial}{\partial u}+\lambda_{1} r_{1}+\lambda_{2} r_{2}+\varphi(u)\right) P_{0}(u, t)=0, \\
& \left(\frac{\partial}{\partial t}+\frac{\partial}{\partial y}+\lambda_{1} r_{1}+\mu_{2}(y)\right) P_{1}(y, t)=0, \\
& \left(\frac{\partial}{\partial t}+\frac{\partial}{\partial x}+\lambda_{2} r_{2}+\mu_{1}(x)\right) P_{2}(x, t)=0,
\end{aligned}
$$$$
\left(\frac{d}{d t}+\lambda_{1} r_{1}+\lambda_{2} r_{2}\right) p_{3}(t)=\int_{0}^{\infty} P_{0}(u, t) \varphi(u) d u,
$$$$
\left(\frac{\partial}{\partial t}+\frac{\partial}{\partial x}+\mu_{1}(x)\right) P_{4}(x, t)=\lambda_{2} r_{2} P_{2}(x, t),
$$$$
\left(\frac{\partial}{\partial t}+\frac{\partial}{\partial z}+\lambda_{1} r_{1}+\alpha(z)\right) P_{5}(z, t)=0,
$$$$
\left(\frac{\partial}{\partial t}+\frac{\partial}{\partial z}+\lambda_{2} r_{2}+\alpha(z)\right) P_{6}(z, t)=0,
$$$$
\left(\frac{\partial}{\partial t}+\frac{\partial}{\partial z}+\alpha(z)\right) P_{7}(z, t)=\lambda_{2} r_{2} P_{6}(z, t)
$$

$$
\begin{aligned}
& \left(\frac{\partial}{\partial t}+\frac{\partial}{\partial y}+\mu_{2}(y)\right) P_{8}(y, t)=\lambda_{1} r_{1} P_{1}(y, t) \\
& \left(\frac{\partial}{\partial t}+\frac{\partial}{\partial x}+\mu_{1}(x)\right) P_{9}(x, t)=0 \\
& \left(\frac{\partial}{\partial t}+\frac{\partial}{\partial z}+\alpha(z)\right) p_{10}(z, t)=\lambda_{1} r_{1} p_{5}(z, t) \\
& \left(\frac{\partial}{\partial t}+\frac{\partial}{\partial y}+\mu_{2}(y)\right) P_{11}(y, t)=0
\end{aligned}
$$

The boundary conditions are:

$$
\begin{aligned}
& P_{0}(t, 0)=\int_{0}^{\infty} P_{1}(y, t) \mu_{2}(y) d y+\int_{0}^{\infty} P_{2}(x, t) \mu_{1}(x) d x+\delta(t) \\
& P_{1}(t, 0)=\int_{0}^{\infty} P_{0}(u, t) \lambda_{2} r_{2} d u+\int_{0}^{\infty} P_{4}(x, t) \mu_{1}(x) d x \\
& +\int_{0}^{\infty} P_{5}(z, t) \alpha(z) d z+\int_{0}^{\infty} P_{9}(x, t) \mu_{1}(x) d x \\
& P_{2}(t, 0)=\int_{0}^{\infty} P_{0}(u, t) \lambda_{1} r_{1} d u+\int_{0}^{\infty} P_{8}(y, t) \mu_{2}(y) d y \\
& +\int_{0}^{\infty} P_{11}(y, t) \mu_{2}(y) d y+\int_{0}^{\infty} P_{6}(z, t) \alpha(z) d z \\
& P_{5}(t, 0)=p_{3}(t) \lambda_{2} r_{2} \\
& P_{6}(t, 0)=p_{3}(t) \lambda_{1} r_{1} \\
& P_{9}(t, 0)=\int_{0}^{\infty} P_{7}(z, t) \alpha(z) d z \\
& P_{11}(t, 0)=\int_{0}^{\infty} P_{10}(z, t) \alpha(z) d z \\
& P_{i}(t, 0)=0, \quad(i=4,7,8,10)
\end{aligned}
$$

Using the following normalizing condition:

$\sum_{i=0}^{2} \int_{0}^{\infty} P_{i}(t, m) d m+p_{3}(t)+\sum_{i=4}^{11} \int_{0}^{\infty} P_{i}(t, m) d m=1$,

$\forall \mathrm{m}=(\mathrm{u}, \mathrm{x}, \mathrm{y}, \mathrm{z})$

Initial conditions are defined as

$$
P_{0}(0, u)=\delta(u)=\left\{\begin{array}{ll}
1 & u=0 \\
0 & u \neq 0
\end{array},\right.
$$


$P_{i}(0, w)=0, \quad w \neq 0, \forall w=(x, y, z)$,

$i=1, \ldots, 11, \quad p_{3}(0)=0$

Taking the limit $t \rightarrow \infty$ in the equations (1) - (21), we obtain the following equations:

$\left(\frac{d}{d u}+\lambda_{1} r_{1}+\lambda_{2} r_{2}+\varphi(u)\right) g_{0}(u)=0$

$\left(\frac{d}{d y}+\lambda_{1} r_{1}+\mu_{2}(y)\right) g_{1}(y)=0$

$\left(\frac{d}{d x}+\lambda_{2} r_{2}+\mu_{1}(x)\right) g_{2}(x)=0$

$\left(\lambda_{1} r_{1}+\lambda_{2} r_{2}\right) p_{3}=\int_{0}^{\infty} g_{0}(u) \varphi(u) d u$

$\left(\frac{d}{d x}+\mu_{1}(x)\right) g_{4}(x)=\lambda_{2} r_{2} g_{2}(x)$ $\left(\frac{d}{d z}+\lambda_{1} r_{1}+\alpha(z)\right) g_{5}(z)=0$

$$
\left(\frac{d}{d z}+\lambda_{2} r_{2}+\alpha(z)\right) g_{6}(z)=0
$$

$\left(\frac{d}{d z}+\alpha(z)\right) g_{7}(z)=\lambda_{2} r_{2} g_{6}(z)$

$\left(\frac{d}{d y}+\mu_{2}(y)\right) g_{8}(y)=\lambda_{1} r_{1} g_{1}(y)$

$\left(\frac{d}{d x}+\mu_{1}(x)\right) g_{9}(x)=0$

$\left(\frac{d}{d z}+\alpha(z)\right) g_{10}(z)=\lambda_{1} r_{1} g_{5}(z)$

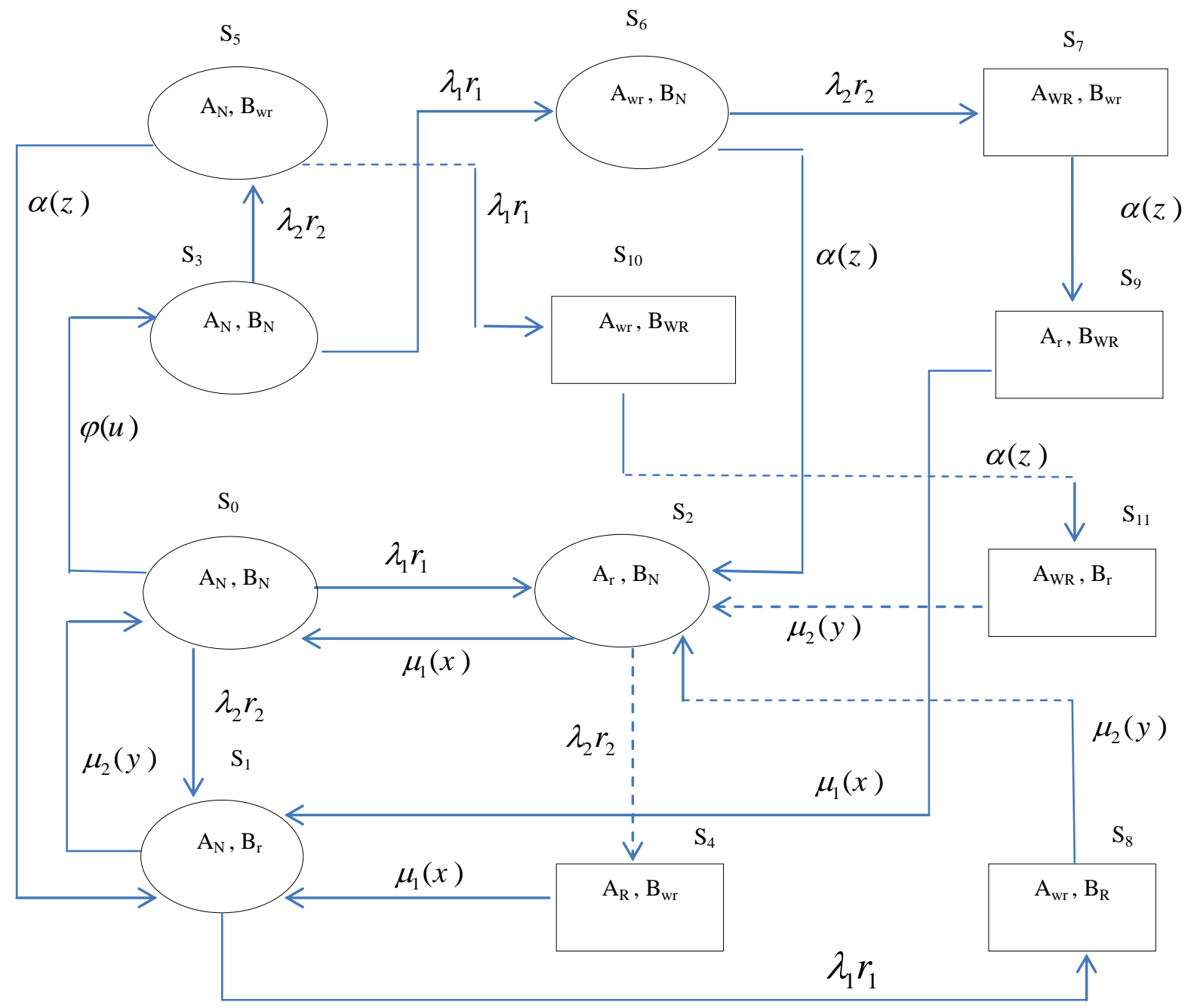

Fig 1: State transition diagram of the system 


$$
\left(\frac{d}{d y}+\mu_{2}(y)\right) g_{11}(z)=0
$$

The boundary conditions are:

$$
\begin{gathered}
g_{0}(0)=\int_{0}^{\infty} g_{1}(y) \mu_{2}(y) d y+\int_{0}^{\infty} g_{2}(x) \mu_{1}(x) d x \\
g_{1}(0)=\int_{0}^{\infty} g_{0}(u) \lambda_{2} r_{2} d u+\int_{0}^{\infty} g_{4}(x) \mu_{1}(x) d x \\
+\int_{0}^{\infty} g_{5}(z) \alpha(z) d z+\int_{0}^{\infty} g_{9}(x) \mu_{1}(x) d x \\
g_{2}(0)=\int_{0}^{\infty} g_{0}(u) \lambda_{1} r_{1} d u+\int_{0}^{\infty} g_{6}(z) \alpha(z) d z \\
+\int_{0}^{\infty} g_{8}(y) \mu_{2}(y) d y+\int_{0}^{\infty} g_{11}(y) \mu_{2}(y) d y
\end{gathered}
$$

$g_{9}(0)=\int_{0}^{\infty} g_{7}(z) \alpha(z) d z, \quad g_{11}(0)=\int_{0}^{\infty} g_{10}(z) \alpha(z) d z$

$g_{5}(0)=p_{3} \lambda_{2} r_{2}, \quad g_{6}(0)=p_{3} \lambda_{1} r_{1}, g_{i}(0)=0$,

$\forall i=4,7,8,10$.

$\sum_{i=0}^{2} \int_{0}^{\infty} g_{i}(m) d m+p_{3}+\sum_{i=4}^{11} \int_{0}^{\infty} g_{i}(m) d m=1$.

$\forall m=(u, x, y, z)$

where, $\mathrm{g}_{i}(m)=\lim _{t \rightarrow \infty} p_{i}(t, m), i=0,1,2,4, \ldots, 11$ which follows the following relations: $p_{i}=\int_{0}^{\infty} g_{i}(m) d m$.

We get the solutions $g_{i}(m), p_{3}$ of the above equations (22) - (38). Using equation (39) we get $p_{0}, \ldots, p_{11}$. (see Appendix A)

Hence, the steady-state availability of the system is

$$
A(\infty)=\sum_{i=0}^{3} p_{i}+\sum_{i=5}^{6} p_{i}
$$

\subsection{The steady-state probability that the} repair man is on vacation is

$P_{r}=\sum_{i=5}^{7} p_{i}+p_{10}$

\subsection{The steady-state probability that the system is waiting for being repaired is:}

$$
P_{w}=p_{7}+p_{10}
$$

\subsection{The steady-state failure frequency is \\ $M=\lambda_{1} r_{1} p_{1}+\lambda_{2} r_{2} p_{2}+\lambda_{1} r_{1} p_{5}+\lambda_{2} r_{2} p_{6}$}

\subsection{Mean time to failure of the system}

\subsubsection{The reliability of the system}

Let the failure states $4,7,8,9,10,11$ be the absorbing states, and then we have another vector Markov process $\left\{\tilde{S}(t), \tilde{X_{1}}(t), \tilde{X_{2}}(t), \tilde{Y_{1}}(t), \tilde{Y_{2}}(t), \mathrm{t} \geq 0\right\}$.

Let: $\pi_{0}(t, u)=\frac{d}{d u} P\left(\tilde{S}(t)=0, \quad \tilde{\mathrm{X}}_{1}(\mathrm{t}) \leq u\right)$,

$\pi_{1}(t, y)=\frac{d}{d y} P\left(\tilde{S}(t)=1, \quad \tilde{\mathrm{Y}}_{2}(\mathrm{t}) \leq y\right)$,

$\pi_{2}(t, x)=\frac{d}{d x} P\left(\tilde{S}(t)=2, \quad \tilde{\mathrm{X}}_{2}(\mathrm{t}) \leq x\right)$

$\pi_{3}(t)=P(\tilde{S}(t)=3)$,

$\pi_{i}(t, z)=\frac{d}{d z} P\left(\tilde{S}(t)=i, \tilde{\mathrm{X}}_{2} \leq z\right), \quad(i=5,6)$

Using the same method in section 4.1, we have the following partial- differential equations:

$\left(\frac{\partial}{\partial t}+\frac{\partial}{\partial u}+\lambda_{1} r_{1}+\lambda_{2} r_{2}+\varphi(u)\right) \pi_{0}(t, u)=0$

$\left(\frac{\partial}{\partial t}+\frac{\partial}{\partial y}+\lambda_{1} r_{1}+\mu_{1}(y)\right) \pi_{1}(t, y)=0$

$\left(\frac{\partial}{\partial t}+\frac{\partial}{\partial x}+\lambda_{2} r_{2}+\mu_{2}(x)\right) \pi_{2}(t, x)=0$

$\left(\frac{d}{d t}+\lambda_{1} r_{1}+\lambda_{2} r_{2}\right) \pi_{3}(t)=\int_{0}^{\infty} \pi_{0}(t, u) \varphi(u) d u$

$\left(\frac{\partial}{\partial t}+\frac{\partial}{\partial z}+\lambda_{1} r_{1}+\alpha(z)\right) \pi_{5}(t, z)=0$

$\left(\frac{\partial}{\partial t}+\frac{\partial}{\partial z}+\lambda_{2} r_{2}+\alpha(z)\right) \pi_{6}(t, z)=0$

The boundary conditions are:

$\pi_{0}(t, 0)=\int_{0}^{\infty} \pi_{1}(t, y) \mu_{1}(y) d y+\int_{0}^{\infty} \pi_{2}(t, x) \mu_{2}(x) d x+\delta t$

$\pi_{1}(t, 0)=\int_{0}^{\infty} \pi_{0}(t, u) \lambda_{2} r_{2} d u+\int_{0}^{\infty} \pi_{5}(t, z) \alpha(z) d z$ 
$\pi_{2}(t, 0)=\int_{0}^{\infty} \pi_{0}(t, u) \lambda_{1} r_{1} d u+\int_{0}^{\infty} \pi_{6}(t, z) \alpha(z) d z$

$$
\pi_{5}(t, 0)=\pi_{3}(t) \lambda_{2} r_{2}, \pi_{6}(t, 0)=\pi_{3}(t) \lambda_{1} r_{1}
$$

The initial conditions are:

$$
\begin{aligned}
& \pi_{0}(0, u)=\delta(u)=\left\{\begin{array}{cc}
1, & \mathrm{u}=0 \\
0, & \mathrm{u} \neq 0
\end{array}, \text { otherwise is } 0\right. \\
& \frac{d}{d u} \pi_{0}^{*}(s, u)+\left(s+\lambda_{1} r_{1}+\lambda_{2} r_{2}+\varphi(u)\right) \pi_{0}^{*}(s, u)=0 \\
& \frac{d}{d y} \pi_{1}^{*}(s, y)+\left(s+\lambda_{1} r_{1}+\mu_{1}(y)\right) \pi_{1}^{*}(s, y)=0 \\
& \frac{d}{d x} \pi_{2}^{*}(s, x)+\left(s+\lambda_{2} r_{2}+\mu_{2}(x)\right) \pi_{2}^{*}(s, x)=0 \\
& \left(s+\lambda_{1} r_{1}+\lambda_{2} r_{2}\right) \pi_{3}^{*}(s)=\int_{0}^{\infty} \pi_{0}^{*}(s, u) \varphi(u) d u \\
& \frac{d}{d z} \pi_{5}^{*}(s, z)+\left(s+\lambda_{1} r_{1}+\alpha(z)\right) \pi_{5}^{*}(s, z)=0 \\
& \frac{d}{d z} \pi_{6}^{*}(s, z)\left(s+\lambda_{2} r_{2}+\alpha(z)\right) \pi_{6}^{*}(s, z)=0 \\
& \pi_{1}^{*}(s, 0)=\int_{0}^{*} \pi_{0}^{*}(s, u) \lambda_{2} r_{2} d u+\int_{0}^{*} \pi_{5}^{*}(s, z) \alpha(z) d z \\
& \pi_{0}^{*}(s, 0)=\int_{0}^{\infty} \pi_{1}^{*}(s, y) \mu_{2}(y) d y+\int_{0}^{\infty} \pi_{2}^{*}(s, x) \mu_{1}(x) d x+1
\end{aligned}
$$

According to the initial conditions, we obtain: $\pi_{3}^{*}(0)=0$.

From the solutions of the equations (54-62), We obtain $\pi_{0}^{*}(s, u), \pi_{1}^{*}(s, y), \pi_{2}^{*}(s, x), \pi_{3}^{*}(s), \pi_{5}^{*}(s, z)$, $\pi_{6}^{*}(s, z)$. ( see Appendix A):

The reliability of the system can be defined as:

$$
\begin{aligned}
R(t) & =\int_{0}^{\infty} \pi_{0}(t, u) d u+\int_{0}^{\infty} \pi_{1}(t, y) d y+\int_{0}^{\infty} \pi_{2}(t, x) d x \\
& +\pi_{3}(t)+\sum_{i=5}^{6} \int_{0}^{\infty} \pi_{i}(t, z) d z .
\end{aligned}
$$

In order to get the reliability of the system, we take The Laplace transformation formula of equation (62):

$$
\begin{aligned}
R^{*}(s) & =\int_{0}^{\infty} \pi_{0}^{*}(s, u) d u+\int_{0}^{\infty} \pi_{1}^{*}(s, y) d y+\int_{0}^{\infty} \pi_{2}^{*}(s, x) d x \\
& +\pi_{3}^{*}(s)+\sum_{i=5}^{6} \int_{0}^{\infty} \pi_{i}^{*}(s, z) d z .
\end{aligned}
$$

4.5.2 The mean time to failure of the system as follows:

$$
M T T F=\int_{0}^{\infty} R(t) d t=\lim _{s \rightarrow 0^{+}} R^{*}(s)
$$

\section{SPECIAL CASES}

In this section, we assume that the Vacation time distribution function is not found, $\varphi(u)=0$,

1. The Vacation time distribution function is not found, $\varphi(u)=0$

2. The state $S_{0}$ is neglected from the system, therefore the transpose from $S_{1}$ and $S_{2}$ move to $S_{3}$

That is mean we have a new system which characterizes by the repairman already on vacation when two units are operating. We can describe the system's equations like the other system.

From these changes and using the same method, we get $p_{0}, \ldots, p_{10}$ and some reliability indices

The steady-state availability is

$A_{s}(\infty)=\sum_{i=1}^{3} p_{i}+\sum_{i=5}^{6} p_{i}$

The steady-state probability that the repair man is on vacation is

$P_{v s}=\sum_{i=5}^{7} p_{i}+p_{10}$

The steady-state probability that the system is waiting for being repaired is

$P_{w s}=P_{7}+P_{10}$

The steady-state failure frequency is

$M_{s}=\lambda_{1} r_{1} p_{1}+\lambda_{2} r_{2} p_{2}+\lambda_{1} r_{1} p_{5}+\lambda_{2} r_{2} p_{6}$

The reliability of the system can be defined as:

$R_{s}^{*}(s)=\sum_{i=1}^{2} \int_{0}^{\infty} L_{i}^{*}(s, x) d x+L_{3}^{*}(s, y)+\sum_{i=5}^{6} \int_{0}^{\infty} L_{i}^{*}(s, z) d z$

The mean time to failure of system as follows:

$\operatorname{MTTF}_{s}=\int_{0}^{\infty} R_{s}(t) d t=\lim _{s \rightarrow 0^{+}} R_{s}^{*}(s)$

Finally, we make comparative analysis between two systems (see Figs. 2 and 3) 


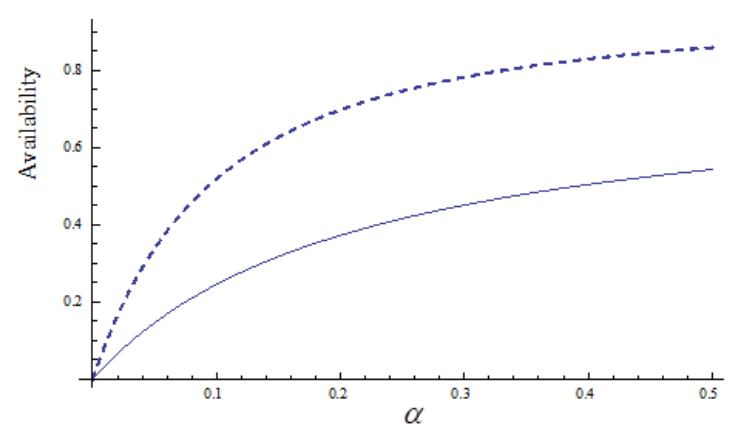

-... System where the repairman has choice between stay or take vacation in initial state

Special case where the repairman is on vacation in initial state

Fig 2: Effect of $\alpha$ on availability

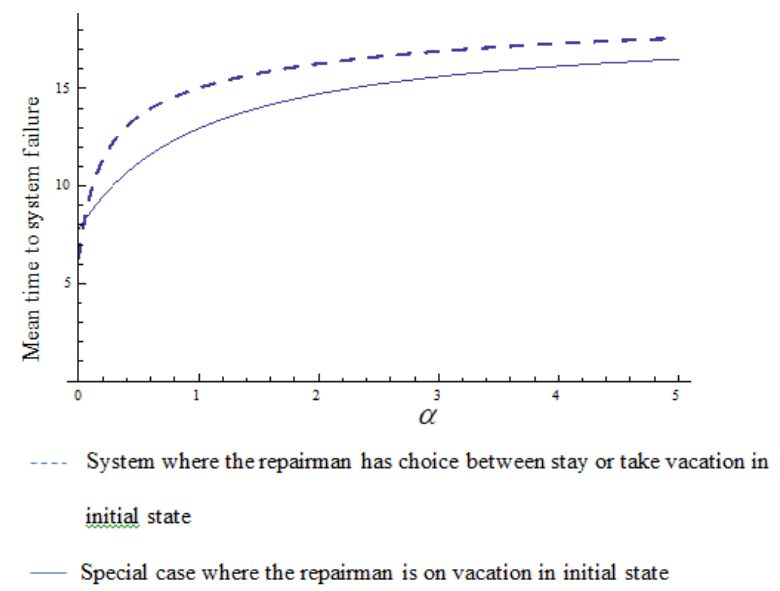

Fig 3: Effect of $\alpha$ on Mean time to system failure

\section{CONCLUSION}

In this paper we studied two repairable systems which influenced by repairman. The repairman has multiple choices either stays or take vacation in initial state, therefore we note the effect of repairman on perform of the system. Explicit expressions for the system availability, MTSF, The steadystate probability that the repair man is on vacation and the steady-state failure frequency for two the systems were derived. After comparative analysis via Figs. 2 and 3, we deduced the system that the repairman stay in initial state is better than the system whose repairman takes vacation in initial state. We recommend that the repairman have to exist in the beginning of the operation.

\section{APPENDIX A}

This section investigates some reliability indices of the system using supplementary variable technique. From equations (22)

-(38) and (39) we get $p_{0}, \ldots, p_{11}$.

$$
\begin{aligned}
& p_{0}=\left(( r _ { 1 } \lambda _ { 1 } + r _ { 2 } \lambda _ { 2 } ) C _ { 0 } \overline { V } _ { 2 } ^ { * } ( r _ { 1 } \lambda _ { 1 } + r _ { 2 } \lambda _ { 2 } ) \left(h_{1}^{*}\left(r_{2} \lambda_{2}\right)\right.\right. \\
& \left.\left.\quad\left(-1+h_{2}^{*}\left(r_{1} \lambda_{1}\right)\right)-h_{2}^{*}\left(r_{1} \lambda_{1}\right)\right)\right) \\
& /\left(\left(r_{1} \lambda_{1}+r_{2} \lambda_{2}\right)\left(-1+r_{1} \lambda_{1} \bar{V}_{2}^{*}\left(r_{1} \lambda_{1}+r_{2} \lambda_{2}\right) h_{1}^{*}\left(r_{2} \lambda_{2}\right)\right)\right. \\
& +h_{1}^{*}\left(r_{2} \lambda_{2}\right)\left(-r_{2} \lambda_{2}\left(-1+v_{1}^{*}\left(r_{1} \lambda_{1}\right)\right)+r_{1} \lambda_{1} v_{1}^{*}\left(r_{2} \lambda_{2}\right)\right) \\
& \left.v_{2}^{*}\left(r_{1} \lambda_{1}+r_{2} \lambda_{2}\right)\right)
\end{aligned}
$$

$$
\begin{aligned}
& p_{1}=C_{0} \bar{H}_{2}^{*}\left(r_{1} \lambda_{1}\right) \\
& p_{2}=C_{0} \bar{H}_{1}^{*}\left(r_{2} \lambda_{2}\right)\left(\left(-r_{1} \lambda_{1}-r_{2} \lambda_{2}\right)(1+(-1\right. \\
& \left.\left.+r_{1} \lambda_{1} \bar{V}_{2}^{*}\left(r_{1} \lambda_{1}+r_{2} \lambda_{2}\right)\right) h_{2}^{*}\left(r_{1} \lambda_{1}\right)\right)-h_{2}^{*}\left(r_{1} \lambda_{1}\right)\left(r_{2} \lambda_{2}\right. \\
& \left.\left.\left(1-v_{1}{ }^{*}\left(r_{1} \lambda_{1}\right)\right)+r_{1} \lambda_{1} v_{1}^{*}\left(r_{2} \lambda_{2}\right)\right) v_{2}^{*}\left(r_{1} \lambda_{1}+r_{2} \lambda_{2}\right)\right) \\
& /\left(( r _ { 1 } \lambda _ { 1 } + r _ { 2 } \lambda _ { 2 } ) \left(-1+r_{1} \lambda_{1} \bar{V}_{2}^{*}\left(r_{1} \lambda_{1}+r_{2} \lambda_{2}\right)\right.\right. \\
& \left(h_{1}^{*}\left(r_{2} \lambda_{2}\right)\right)+h_{1}^{*}\left(r_{2} \lambda_{2}\right)\left(r_{2} \lambda_{2}\left(1-v_{1}^{*}\left(r_{1} \lambda_{1}\right)\right)\right. \\
& \left.\left.+r_{1} \lambda_{1} v_{1}^{*}\left(r_{2} \lambda_{2}\right)\right) v_{2}^{*}\left(r_{1} \lambda_{1}+r_{2} \lambda_{2}\right)\right) \\
& p_{3}=C_{0}\left(h_{1}^{*}\left(r_{2} \lambda_{2}\right)\left(-1+h_{2}^{*}\left(r_{1} \lambda_{1}\right)\right)-h_{2}^{*}\left(r_{1} \lambda_{1}\right)\right) v_{2}^{*}\left(r_{1} \lambda_{1}\right. \\
& \left.+r_{2} \lambda_{2}\right) /\left(( r _ { 1 } \lambda _ { 1 } + r _ { 2 } \lambda _ { 2 } ) \left(-1+r_{1} \lambda_{1} \bar{V}_{2}^{*}\left(r_{1} \lambda_{1}+r_{2} \lambda_{2}\right)\right.\right. \\
& \left.h_{1}^{*}\left(r_{2} \lambda_{2}\right)\right)+h_{1}^{*}\left(r_{2} \lambda_{2}\right)\left(-r_{2} \lambda_{2}\left(-1+v_{1}^{*}\left(r_{1} \lambda_{1}\right)\right)+r_{1} \lambda_{1} v_{1}^{*}\right. \\
& \left.\left.\left(r_{2} \lambda_{2}\right)\right) v_{2}^{*}\left(r_{1} \lambda_{1}+r_{2} \lambda_{2}\right)\right) \\
& p_{4}=C_{0}\left(-1+\mu_{1} \bar{H}_{1}^{*}\left(r_{2} \lambda_{2}\right)\right)\left(\left(r_{1} \lambda_{1}+r_{2} \lambda_{2}\right)(1+(-1\right. \\
& \left.\left.+r_{1} \lambda_{1} \bar{V}_{2}^{*}\left(r_{1} \lambda_{1}+r_{2} \lambda_{2}\right)\right) h_{2}^{*}\left(r_{1} \lambda_{1}\right)\right)+h_{2}^{*}\left(r_{1} \lambda_{1}\right)\left(-r_{2} \lambda_{2}\right. \\
& \left.\left.\left(-1+v_{1}^{*}\left(r_{1} \lambda_{1}\right)\right)+r_{1} \lambda_{1} v_{1}^{*}\left(r_{2} \lambda_{2}\right)\right) v_{2}^{*}\left(r_{1} \lambda_{1}+r_{2} \lambda_{2}\right)\right) \\
& / \mu_{1}\left(( r _ { 1 } \lambda _ { 1 } + r _ { 2 } \lambda _ { 2 } ) \left(-1+r_{1} \lambda_{1} \bar{V}_{2}^{*}\left(r_{1} \lambda_{1}+r_{2} \lambda_{2}\right)\right.\right. \\
& \left.h_{1}^{*}\left(r_{2} \lambda_{2}\right)\right)+h_{1}^{*}\left(r_{2} \lambda_{2}\right)\left(-r_{2} \lambda_{2}\left(-1+v_{1}^{*}\left(r_{1} \lambda_{1}\right)\right)\right. \\
& \left.\left.+r_{1} \lambda_{1} v_{1}^{*}\left(r_{2} \lambda_{2}\right)\right) v_{2}^{*}\left(r_{1} \lambda_{1}+r_{2} \lambda_{2}\right)\right) \\
& p_{5}=r_{2} \lambda_{2} C_{0} \bar{V}_{1}^{*}\left(r_{1} \lambda_{1}\right)\left(-h_{1}^{*}\left(r_{2} \lambda_{2}\right)\left(-1+h_{2}^{*}\left(r_{1} \lambda_{1}\right)\right)+h_{2}^{*}\left(r_{1} \lambda_{1}\right)\right) \\
& v_{2}^{*}\left(r_{1} \lambda_{1}+r_{2} \lambda_{2}\right) /\left(-\left(r_{1} \lambda_{1}+r_{2} \lambda_{2}\right)+r_{1} \lambda_{1} \bar{V}_{2}^{*}\left(r_{1} \lambda_{1}+r_{2} \lambda_{2}\right)\right. \\
& \left(-1 h_{1}^{*}\left(r_{2} \lambda_{2}\right)\right)+h_{1}^{*}\left(r_{2} \lambda_{2}\right)\left(r_{2} \lambda_{2}\left(-1+v_{1}^{*}\left(r_{1} \lambda_{1}\right)\right)\right. \\
& \left.\left.-r_{1} \lambda_{1} v_{1}^{*}\left(r_{2} \lambda_{2}\right)\right) v_{2}^{*}\left(r_{1} \lambda_{1}+r_{2} \lambda_{2}\right)\right) \\
& p_{6}=r_{1} \lambda_{1} C_{0} \bar{V}_{1}^{*}\left(r_{2} \lambda_{2}\right) v_{2}^{*}\left(r_{1} \lambda_{1}+r_{2} \lambda_{2}\right)\left(h_{1}^{*}\left(r_{2} \lambda_{2}\right)\right. \\
& \left.\left(-1+h_{2}^{*}\left(r_{1} \lambda_{1}\right)\right)-h_{2}^{*}\left(r_{1} \lambda_{1}\right)\right) /\left(\left(r_{1} \lambda_{1}+r_{2} \lambda_{2}\right)\right. \\
& \left(-1+r_{1} \lambda_{1} \bar{V}_{2}^{*}\left(r_{1} \lambda_{1}+r_{2} \lambda_{2}\right) h_{1}^{*}\left(r_{2} \lambda_{2}\right)\right)+h_{1}^{*}\left(r_{2} \lambda_{2}\right) \\
& \left(-r_{2} \lambda_{2}\left(-1+v_{1}^{*}\left(r_{1} \lambda_{1}\right)\right)+r_{1} \lambda_{1} v_{1}^{*}\left(r_{2} \lambda_{2}\right) v_{2}^{*}\left(r_{1} \lambda_{1}+r_{2} \lambda_{2}\right)\right) \\
& p_{7}=-r_{1} \lambda_{1} C_{0}\left(-1+\alpha_{1} \bar{V}_{1}^{*}\left(r_{2} \lambda_{2}\right)\right)\left(h_{1}^{*}\left(r_{2} \lambda_{2}\right)(-1\right. \\
& \left.\left.+h_{2}^{*}\left(r_{1} \lambda_{1}\right)\right)-h_{2}^{*}\left(r_{1} \lambda_{1}\right)\right) v_{2}^{*}\left(r_{1} \lambda_{1}+r_{2} \lambda_{2}\right) \\
& / \alpha_{1}\left(\left(r_{1} \lambda_{1}+r_{2} \lambda_{2}\right)\left(-1+r_{1} \lambda_{1} \bar{V}_{2}^{*}\left(r_{1} \lambda_{1}+r_{2} \lambda_{2}\right) h_{1}^{*}\left(r_{2} \lambda_{2}\right)\right)\right. \\
& +h_{1}^{*}\left(r_{2} \lambda_{2}\right)\left(-r_{2} \lambda_{2}\left(-1+v_{1}^{*}\left(r_{1} \lambda_{1}\right)\right)+r_{1} \lambda_{1} v_{1}^{*}\left(r_{2} \lambda_{2}\right)\right) \\
& \left.v_{2}^{*}\left(r_{1} \lambda_{1}+r_{2} \lambda_{2}\right)\right)
\end{aligned}
$$




$$
\begin{aligned}
& p_{8}=C_{0}\left(\frac{1}{\mu_{2}}-\bar{H}_{2}^{*}\left(r_{1} \lambda_{1}\right)\right) \\
& p_{9}=-r_{1} \lambda_{1} C_{0}\left(h_{1}^{*}\left(r_{2} \lambda_{2}\right)\left(-1+h_{2}^{*}\left(r_{1} \lambda_{1}\right)\right)-h_{2}^{*}\left(r_{1} \lambda_{1}\right)\right)(-1 \\
& \left.+v_{1}^{*}\left(r_{2} \lambda_{2}\right)\right) v_{2}^{*}\left(r_{1} \lambda_{1}+r_{2} \lambda_{2}\right) / \mu_{1}\left(( r _ { 1 } \lambda _ { 1 } + r _ { 2 } \lambda _ { 2 } ) \left(-1+r_{1} \lambda_{1}\right.\right. \\
& \left.\bar{V}_{2}^{*}\left(r_{1} \lambda_{1}+r_{2} \lambda_{2}\right) h_{1}^{*}\left(r_{2} \lambda_{2}\right)\right)+h_{1}^{*}\left(r_{2} \lambda_{2}\right)\left(-r_{2} \lambda_{2}(-1\right. \\
& \left.\left.\left.+v_{1}^{*}\left(r_{1} \lambda_{1}\right)\right)+r_{1} \lambda_{1} v_{1}^{*}\left(r_{2} \lambda_{2}\right)\right) v_{2}^{*}\left(r_{1} \lambda_{1}+r_{2} \lambda_{2}\right)\right) \\
& p_{10}=-r_{2} \lambda_{2} C_{0}\left(-1+\alpha_{1} \bar{V}_{1}^{*}\left(r_{1} \lambda_{1}\right)\right)\left(h_{1}^{*}\left(r_{2} \lambda_{2}\right)(-1\right. \\
& \left.\left.+h_{2}^{*}\left(r_{1} \lambda_{1}\right)\right)-h_{2}^{*}\left(r_{1} \lambda_{1}\right)\right) v_{2}^{*}\left(r_{1} \lambda_{1}+r_{2} \lambda_{2}\right) / \alpha_{1}\left(\left(r_{1} \lambda_{1}\right.\right. \\
& \left.+r_{2} \lambda_{2}\right)\left(-1+r_{1} \lambda_{1} \bar{V}_{2}^{*}\left(r_{1} \lambda_{1}+r_{2} \lambda_{2}\right) h_{1}^{*}\left(r_{2} \lambda_{2}\right)\right)+h_{1}^{*}\left(r_{2} \lambda_{2}\right) \\
& \left.\left(-r_{2} \lambda_{2}\left(-1+v_{1}^{*}\left(r_{1} \lambda_{1}\right)\right)+r_{1} \lambda_{1} v_{1}^{*}\left(r_{2} \lambda_{2}\right)\right) v_{2}^{*}\left(r_{1} \lambda_{1}+r_{2} \lambda_{2}\right)\right) \\
& p_{11}=-r_{2} \lambda_{2} C_{0}\left(h_{1}^{*}\left(r_{2} \lambda_{2}\right)\left(-1+h_{2}^{*}\left(r_{1} \lambda_{1}\right)\right)-h_{2}^{*}\left(r_{1} \lambda_{1}\right)\right) \\
& \left(-1+v_{1}^{*}\left(r_{1} \lambda_{1}\right)\right) v_{2}^{*}\left(r_{1} \lambda_{1}+r_{2} \lambda_{2}\right) / \mu_{2}\left(\left(r_{1} \lambda_{1}+r_{2} \lambda_{2}\right)(-1\right. \\
& \left.+r_{1} \lambda_{1} \bar{V}_{2}^{*}\left(r_{1} \lambda_{1}+r_{2} \lambda_{2}\right) h_{1}^{*}\left(r_{2} \lambda_{2}\right)\right)+h_{1}^{*}\left(r_{2} \lambda_{2}\right)\left(-r_{2} \lambda_{2}(-1\right. \\
& \left.\left.\left.+v_{1}^{*}\left(r_{1} \lambda_{1}\right)\right)+r_{1} \lambda_{1} v_{1}^{*}\left(r_{2} \lambda_{2}\right)\right) v_{2}^{*}\left(r_{1} \lambda_{1}+r_{2} \lambda_{2}\right)\right) \\
& C_{0}=\left\{\left(r_{1} \lambda_{1}+r_{2} \lambda_{2}\right)\left(-1+r_{1} \lambda_{1} \bar{V}_{2}^{*}\left(r_{1} \lambda_{1}+r_{2} \lambda_{2}\right) h_{1}^{*}\left(r_{2} \lambda_{2}\right)\right)\right. \\
& +h_{1}^{*}\left(r_{2} \lambda_{2}\right)\left(-r_{2} \lambda_{2}\left(-1+v_{1}^{*}\left(r_{1} \lambda_{1}\right)+r_{1} \lambda_{1} v_{1}^{*}\left(r_{2} \lambda_{2}\right)\right)\right. \\
& \left.v_{2}^{*}\left(r_{1} \lambda_{1}+r_{2} \lambda_{2}\right)\right\} /\left\{\frac { 1 } { \mu _ { 1 } \mu _ { 2 } } ( r _ { 1 } \lambda _ { 1 } + r _ { 2 } \lambda _ { 2 } ) \left(-\mu_{2}+\mu_{1}(-1\right.\right. \\
& \left.+\left(r_{1} \lambda_{1}-\mu_{2}\right) V_{2}^{*}\left(r_{1} \lambda_{1}+r_{2} \lambda_{2}\right) h_{1}^{*}\left(r_{2} \lambda_{2}\right)\right)+\mu_{2}(1 \\
& \left.\left.-\bar{V}_{2}^{*}\left(r_{1} \lambda_{1}+r_{2} \lambda_{2}\right)\left(r_{1} \lambda_{1}-\mu_{1}\left(-1+h_{1}^{*}\left(r_{2} \lambda_{2}\right)\right)\right)\right) h_{2}^{*}\left(r_{1} \lambda_{1}\right)\right) \\
& -\left(-\frac{r_{2} \lambda_{2} h_{2}^{*}\left(r_{1} \lambda_{1}\right)\left(-1+v_{1}^{*}\left(r_{1} \lambda_{1}\right)\right)}{\mu_{1}}+\left(1-h_{1}^{*}\left(r_{2} \lambda_{2}\right)\right) h_{2}^{*}\left(r_{1} \lambda_{1}\right)\right. \\
& \left(r_{1} \lambda_{1}\left(\frac{1}{\alpha_{1}}+\frac{1}{\mu_{1}}\right)+\frac{\alpha_{1} \mu_{2}+r_{2} \lambda_{2}\left(\alpha_{1}+\mu_{2}-\alpha_{1} v_{2}^{*}\left(r_{1} \lambda_{1}\right)\right)}{\alpha_{1} \mu_{2}}\right) \\
& +\frac{h_{1}^{*}\left(r_{2} \lambda_{2}\right)\left(\alpha_{1}+r_{2} \lambda_{2}\right) \mu_{1}}{\alpha_{1} \mu_{1}}+\frac{h_{1}^{*}\left(r_{2} \lambda_{2}\right) r_{1} \lambda_{1}\left(\mu_{1}-\alpha_{1}\left(-1+v_{1}^{*}\left(r_{2} \lambda_{2}\right)\right)\right)}{\alpha_{1} \mu_{1}} \\
& \left.\left.\left.+r_{1} \lambda_{1} h_{1}^{*}\left(r_{2} \lambda_{2}\right)\left(-\frac{1}{\mu_{2}}+\frac{h_{2}^{*}\left(r_{1} \lambda_{1}\right)}{\mu_{1}}\right) v_{1}^{*}\left(r_{2} \lambda_{2}\right)\right) v_{2}^{*}\left(r_{1} \lambda_{1}+r_{2} \lambda_{2}\right)\right)\right\}
\end{aligned}
$$

\section{The steady-state availability of the system is}

$$
A(\infty)=\sum_{i=0}^{3} p_{i}+\sum_{i=5}^{6} p_{i}
$$

$A(\infty)=\left(C_{0}\left(\left(h_{1}^{*}\left(r_{2} \lambda_{2}\right)\left(-1+h_{2}^{*}\left(r_{1} \lambda_{1}\right)\right)-h_{2}^{*}\left(r_{1} \lambda_{1}\right)\right) v_{2}^{*}\left(r_{1} \lambda_{1}\right.\right.\right.$

$\left.+r_{2} \lambda_{2}\right)+r_{1}^{2} \lambda_{1}^{2} \bar{V}_{2}^{*}\left(r_{1} \lambda_{1}+r_{2} \lambda_{2}\right)\left(-h_{2}^{*}\left(r_{1} \lambda_{1}\right) \bar{H}_{1}^{*}\left(r_{2} \lambda_{2}\right)\right.$ $\left.+h_{1}^{*}\left(r_{2} \lambda_{2}\right) \bar{H}_{2}^{*}\left(r_{1} \lambda_{1}\right)\right)+r_{2} \lambda_{2}\left(\bar{V}_{2}^{*}\left(r_{1} \lambda_{1}+r_{2} \lambda_{2}\right)\right.$

$\left(h_{1}^{*}\left(r_{2} \lambda_{2}\right)\left(-1+h_{2}^{*}\left(r_{1} \lambda_{1}\right)\right)-h_{2}^{*}\left(r_{1} \lambda_{1}\right)\right)+\bar{V}_{1}^{*}\left(r_{1} \lambda_{1}\right)$

$\left(-h_{1}^{*}\left(r_{2} \lambda_{2}\right)+\left(-1+h_{1}^{*}\left(r_{2} \lambda_{2}\right)\right) h_{2}^{*}\left(r_{1} \lambda_{1}\right)\right) v_{2}^{*}\left(r_{1} \lambda_{1}\right.$ $\left.+r_{2} \lambda_{2}\right)+\left(-1+h_{2}^{*}\left(r_{1} \lambda_{1}\right)\left(1+\left(-1+v_{1}^{*}\left(r_{1} \lambda_{1}\right)\right) v_{2}^{*}\left(r_{1} \lambda_{1}\right.\right.\right.$ $\left.\left.\left.+r_{2} \lambda_{2}\right)\right)\right) \bar{H}_{1}^{*}\left(r_{2} \lambda_{2}\right)+\left(-1-h_{1}^{*}\left(r_{2} \lambda_{2}\right)\left(-1+v_{1}^{*}\left(r_{1} \lambda_{1}\right)\right)\right.$ $\left.\left.v_{2}{ }^{*}\left(r_{1} \lambda_{1}+r_{2} \lambda_{2}\right)\right) \bar{H}_{2}^{*}\left(r_{1} \lambda_{1}\right)\right)+r_{1} \lambda_{1}\left(\bar{V}_{1}^{*}\left(r_{2} \lambda_{2}\right)\left(-h_{1}^{*}\left(r_{2} \lambda_{2}\right)\right.\right.$ $\left.+\left(-1+h_{1}^{*}\left(r_{2} \lambda_{2}\right)\right) h_{2}^{*}\left(r_{1} \lambda_{1}\right)\right) v_{2}^{*}\left(r_{1} \lambda_{1}+r_{2} \lambda_{2}\right)+(-1$

$\left.+h_{2}^{*}\left(r_{1} \lambda_{1}\right)\left(1-v_{1}^{*}\left(r_{2} \lambda_{2}\right) v_{2}^{*}\left(r_{1} \lambda_{1}+r_{2} \lambda_{2}\right)\right)\right) \bar{H}_{1}^{*}\left(r_{2} \lambda_{2}\right)+(-1$ $\left.+h_{1}^{*}\left(r_{2} \lambda_{2}\right) v_{1}^{*}\left(r_{2} \lambda_{2}\right) v_{2}^{*}\left(r_{1} \lambda_{1}+r_{2} \lambda_{2}\right)\right) \bar{H}_{2}^{*}\left(r_{1} \lambda_{1}\right)+\bar{V}_{2}^{*}\left(r_{1} \lambda_{1}\right.$ $\left.+r_{2} \lambda_{2}\right)\left(-h_{2}^{*}\left(r_{1} \lambda_{1}\right)\left(1+r_{2} \lambda_{2} \bar{H}_{1}^{*}\left(r_{2} \lambda_{2}\right)\right)+h_{1}^{*}\left(r_{2} \lambda_{2}\right)(-1\right.$ $\left.\left.\left.\left.\left.+h_{2}^{*}\left(r_{1} \lambda_{1}\right)+r_{2} \lambda_{2} \bar{H}_{2}^{*}\left(r_{1} \lambda_{1}\right)\right)\right)\right)\right)\right) /\left\{\left(\left(r_{1} \lambda_{1}+r_{2} \lambda_{2}\right)\left(-1+r_{1} \lambda_{1}\right.\right.\right.$ $\left.\bar{V}_{2}^{*}\left(r_{1} \lambda_{1}+r_{2} \lambda_{2}\right) h_{1}^{*}\left(r_{2} \lambda_{2}\right)\right)+h_{1}^{*}\left(r_{2} \lambda_{2}\right)\left(-r_{2} \lambda_{2}(-1\right.$ $\left.\left.\left.+v_{1}^{*}\left(r_{1} \lambda_{1}\right)\right)+r_{1} \lambda_{1} v_{1}^{*}\left(r_{2} \lambda_{2}\right)\right) v_{2}^{*}\left(r_{1} \lambda_{1}+r_{2} \lambda_{2}\right)\right\}$

Taking the Laplace transform with respect to $t$ to equations (54 - 62), we get

$$
\begin{aligned}
& \pi_{0}^{*}(\mathrm{~s}, \mathrm{u})=\mathrm{e}^{-u\left(s+r_{1} \lambda_{1}+r_{2} \lambda_{2}\right)} \pi_{0}^{*}(s, 0) \bar{V}_{2}(u), \\
& \pi_{1}^{*}(\mathrm{~s}, \mathrm{y})=\mathrm{e}^{-y\left(s+r_{1} \lambda_{1}\right)} \pi_{1}^{*}(s, 0) \bar{H}_{2}(y), \\
& \pi_{2}^{*}(\mathrm{~s}, \mathrm{X})=\mathrm{e}^{-x\left(s+r_{2} \lambda_{2}\right)} \pi_{2}^{*}(s, 0) \bar{H}_{1}(x), \\
& \pi_{3}^{*}(s)=\frac{\pi_{0}^{*}(s, 0) v_{2}\left(s+r_{1} \lambda_{1}+r_{2} \lambda_{2}\right)}{s+r_{1} \lambda_{1}+r_{2} \lambda_{2}} \\
& \pi_{5}^{*}(s, z)=\mathrm{e}^{-z\left(s+r_{1} \lambda_{1}\right)} \pi_{5}^{*}(s, 0) \overline{V_{1}}(z), \\
& \pi_{6}^{*}(\mathrm{~s}, \mathrm{z})=\mathrm{e}^{-z\left(s+r_{2} \lambda_{2}\right)} \pi_{6}^{*}(s, 0) \overline{V_{1}}(z) \\
& \pi_{0}^{*}(s, 0)=\left(\left(s+r_{1} \lambda_{1}+r_{2} \lambda_{2}\right)\left(1+C_{1}(s) h_{2}^{*}\left(s+r_{1} \lambda_{1}\right)\right)\right) \\
& /\left(s+r_{2} \lambda_{2}+r_{1} \lambda_{1}\left(1-\left(v_{1}\left(s+r_{2} \lambda_{2}\right) v_{2}\left(s+r_{1} \lambda_{1}+r_{2} \lambda_{2}\right)\right.\right.\right. \\
& \left.+\left(s+r_{1} \lambda_{1}+r_{2} \lambda_{2}\right) \overline{V_{2}}\left(s+r_{1} \lambda_{1}+r_{2} \lambda_{2}\right)\right) h_{1}{ }^{*}\left(s+r_{2} \lambda_{2}\right)(1 \\
& \left.\left.\left.+C_{1}(s) h_{2}^{*}\left(s+r_{1} \lambda_{1}\right)\right)\right)\right) \\
& \pi_{1}^{*}(s, 0)=\left(\left(s+r_{1} \lambda_{1}+r_{2} \lambda_{2}\right) C_{1}(s)\right) /\left(s+r_{2} \lambda_{2}+r_{1} \lambda_{1}\right. \\
& \left(1-\left(v_{1}\left(s+r_{2} \lambda_{2}\right) v_{2}\left(s+r_{1} \lambda_{1}+r_{2} \lambda_{2}\right)+\left(s+r_{1} \lambda_{1}+r_{2} \lambda_{2}\right)\right.\right. \\
& \left.\left.\left.\overline{V_{2}}\left(s+r_{1} \lambda_{1}+r_{2} \lambda_{2}\right)\right) h_{1}{ }^{*}\left(s+r_{2} \lambda_{2}\right)\left(1+C_{1}(s) h_{2}{ }^{*}\left(s+r_{1} \lambda_{1}\right)\right)\right)\right) \\
& \pi_{2}^{*}(s, 0)=\left(r _ { 1 } \lambda _ { 1 } \left(-\frac{v_{1}\left(s+r_{2} \lambda_{2}\right) v_{2}\left(s+r_{1} \lambda_{1}+r_{2} \lambda_{2}\right)}{s+r_{1} \lambda_{1}+r_{2} \lambda_{2}}\right.\right. \\
& \left.\left.-\bar{V}_{2}\left(s+r_{1} \lambda_{1}+r_{2} \lambda_{2}\right)\right)\left(1+C_{1}(s) h_{2}^{*}\left(s+r_{1} \lambda_{1}\right)\right)\right)
\end{aligned}
$$




$$
\begin{aligned}
& /\left(-1+\frac{1}{s+r_{1} \lambda_{1}+r_{2} \lambda_{2}} r_{1} \lambda_{1}\left(v_{1}\left(s+r_{2} \lambda_{2}\right) v_{2}\left(s+r_{1} \lambda_{1}+r_{2} \lambda_{2}\right)\right.\right. \\
& \left.+\left(s+r_{1} \lambda_{1}+r_{2} \lambda_{2}\right) \bar{V}_{2}\left(s+r_{1} \lambda_{1}+r_{2} \lambda_{2}\right)\right) h_{1}^{*}\left(s+r_{2} \lambda_{2}\right) \\
& \left.\left(1+C_{0}(s) h_{2}^{*}\left(s+r_{1} \lambda_{1}\right)\right)\right) \\
& \pi_{3}^{*}(s, 0)=\left(v_{2}\left(s+r_{1} \lambda_{1}+r_{2} \lambda_{2}\right)\left(1+C_{1}(s) h_{2}^{*}\left(s+r_{1} \lambda_{1}\right)\right)\right) \\
& /\left(s+r_{2} \lambda_{2}+r_{1} \lambda_{1}\left(1-\left(v_{1}\left(s+r_{2} \lambda_{2}\right)\right.\right.\right. \\
& \left.v_{2}\left(s+r_{1} \lambda_{1}+r_{2} \lambda_{2}\right)+\left(s+r_{1} \lambda_{1}+r_{2} \lambda_{2}\right) \overline{V_{2}}\left(s+r_{1} \lambda_{1}+r_{2} \lambda_{2}\right)\right) \\
& \left.\left.h_{1}^{*}\left(s+r_{2} \lambda_{2}\right)\left(1+C_{1}(s) h_{2}^{*}\left(s+r_{1} \lambda_{1}\right)\right)\right)\right) \\
& \pi_{5}^{*}(s, 0)=\left(r_{2} \lambda_{2} v_{2}\left(s+r_{1} \lambda_{1}+r_{2} \lambda_{2}\right)\left(1+C_{1}(s) h_{1}^{*}\left(s+r_{1} \lambda_{1}\right)\right)\right) \\
& /\left(s+r_{2} \lambda_{2}+r_{1} \lambda_{1}\left(1-\left(v_{1}\left(s+r_{2} \lambda_{2}\right) v_{2}\left(s+r_{1} \lambda_{1}+r_{2} \lambda_{2}\right)\right.\right.\right. \\
& \left.+\left(s+r_{1} \lambda_{1}+r_{2} \lambda_{2}\right) \overline{V_{2}}\left(s+r_{1} \lambda_{1}+r_{2} \lambda_{2}\right)\right)\left(1+C_{1}(s) h_{1}^{*}(s\right. \\
& \left.\left.\left.\left.+r_{1} \lambda_{1}\right)\right) h_{2}^{*}\left(s+r_{2} \lambda_{2}\right)\right)\right) \\
& \pi_{6}^{*}(s, 0)=\left(r _ { 1 } \lambda _ { 1 } v _ { 2 } ( s + r _ { 1 } \lambda _ { 1 } + r _ { 2 } \lambda _ { 2 } ) \left(1+C_{1}(s) h_{1}^{*}(s\right.\right. \\
& \left.\left.\left.+r_{1} \lambda_{1}\right)\right)\right) /\left(s+r_{2} \lambda_{2}+r_{1} \lambda_{1}\left(1-\left(v _ { 1 } ( s + r _ { 2 } \lambda _ { 2 } ) v _ { 2 } \left(s+r_{1} \lambda_{1}\right.\right.\right.\right. \\
& \left.\left.+r_{2} \lambda_{2}\right)+\left(s+r_{1} \lambda_{1}+r_{2} \lambda_{2}\right) \overline{V_{2}}\left(s+r_{1} \lambda_{1}+r_{2} \lambda_{2}\right)\right)\left(1+C_{1}(s)\right. \\
& \left.\left.\left.h_{1}^{*}\left(s+r_{1} \lambda_{1}\right)\right) h_{2}{ }^{*}\left(s+r_{2} \lambda_{2}\right)\right)\right) \\
& C_{1}(s)=\left(r _ { 2 } \lambda _ { 2 } \left(v_{1}\left(s+r_{1} \lambda_{1}\right) v_{2}\left(s+r_{1} \lambda_{1}+r_{2} \lambda_{2}\right)\right.\right. \\
& \left.\left.+\left(s+r_{1} \lambda_{1}+r_{2} \lambda_{2}\right) \overline{V_{2}}\left(s+r_{1} \lambda_{1}+r_{2} \lambda_{2}\right)\right)\right) \\
& \left.+r_{2} \lambda_{2}-r_{2} \lambda_{2}\left(v_{1}\left(s+r_{1} \lambda_{1}\right) v_{2}\left(s+r_{1} \lambda_{1}+r_{2} \lambda_{2}\right) \bar{V}_{2}\left(s+r_{1} \lambda_{1}+r_{2} \lambda_{2}\right)\right) h_{2}^{*}\left(s+r_{1} \lambda_{1}\right)\right) \\
& { }^{*}(s)
\end{aligned}
$$

\section{The reliability of the system can be defined as:}

$$
\begin{aligned}
& R(t)=\int_{0}^{\infty} \pi_{0}(t, u) d u+\int_{0}^{\infty} \pi_{1}(t, y) d y+\int_{0}^{\infty} \pi_{2}(t, x) d x \\
& +\pi_{3}(t)+\sum_{i=5}^{6} \int_{0}^{\infty} \pi_{i}(t, z) d z .
\end{aligned}
$$

The Laplace transformation formula of the system is:

$$
\begin{aligned}
& R^{*}(s)=\int_{0}^{\infty} \pi_{0}^{*}(s, u) d u+\int_{0}^{\infty} \pi_{1}^{*}(s, y) d y+\int_{0}^{\infty} \pi_{2}^{*}(s, x) d x \\
& +\pi_{3}^{*}(s)+\sum_{i=5}^{6} \int_{0}^{\infty} \pi_{i}^{*}(s, z) d z . \\
& R^{*}(s)=\left(v _ { 2 } ^ { * } ( s + r _ { 1 } \lambda _ { 1 } + r _ { 2 } \lambda _ { 2 } ) \left(1+r_{2} \lambda_{2} \bar{V}_{1}^{*}\left(s+r_{1} \lambda_{1}\right)\right.\right. \\
& \left.+r_{1} \lambda_{1}\left(v_{1}^{*}\left(s+r_{2} \lambda_{2}\right) \bar{H}_{2}^{*}\left(s+r_{2} \lambda_{2}\right)+\bar{V}_{1}^{*}\left(s+r_{2} \lambda_{2}\right)\right)\right) \\
& \left(1+C_{1}(s) h_{2}^{*}\left(s+r_{1} \lambda_{1}\right)\right)+\left(s+r_{1} \lambda_{1}+r_{2} \lambda_{2}\right)\left(\left(1+r_{1} \lambda_{1} \bar{H}_{2}^{*}(s\right.\right. \\
& \left.\left.+r_{2} \lambda_{2}\right)\right) \bar{V}_{2}^{*}\left(s+r_{1} \lambda_{1}+r_{2} \lambda_{2}\right)+C_{1}(s)\left(\bar{H}_{1}^{*}\left(s+r_{1} \lambda_{1}\right)\right.
\end{aligned}
$$

$$
\begin{aligned}
& \left.\left.\left.+\left(1+r_{1} \lambda_{1} \bar{H}_{2}^{*}\left(s+r_{2} \lambda_{2}\right)\right) \bar{V}_{2}^{*}\left(s+r_{1} \lambda_{1}+r_{2} \lambda_{2}\right) h_{2}^{*}\left(s+r_{1} \lambda_{1}\right)\right)\right)\right) \\
& /\left(s+r_{2} \lambda_{2}+r_{1} \lambda_{1}\left(1-\left(v_{1}^{*}\left(s+r_{2} \lambda_{2}\right) v_{2}^{*}\left(s+r_{1} \lambda_{1}+r_{2} \lambda_{2}\right)\right.\right.\right. \\
& \left.+\left(s+r_{1} \lambda_{1}+r_{2} \lambda_{2}\right) \bar{V}_{2}^{*}\left(s+r_{1} \lambda_{1}+r_{2} \lambda_{2}\right)\right) h_{1}^{*}\left(s+r_{2} \lambda_{2}\right) \\
& \left.\left.\left(1+C_{1}(s) h_{2}^{*}\left(s+r_{1} \lambda_{1}\right)\right)\right)\right)
\end{aligned}
$$

The mean time to failure of the system as follow

$$
\begin{aligned}
& M T T F=\int_{0}^{\infty} R(t) d t=\lim _{s \rightarrow 0^{+}} R^{*}(s) \\
& \operatorname{MTTF}=-\left(v _ { 2 } ( r _ { 1 } \lambda _ { 1 } + r _ { 2 } \lambda _ { 2 } ) \left(1+r_{2} \lambda_{2}\left(v_{1}\left(r_{1} \lambda_{1}\right) \bar{H}_{1}\left(r_{1} \lambda_{1}\right)\right.\right.\right. \\
& \left.\left.+\overline{V_{1}}\left(r_{1} \lambda_{1}\right)\right)+r_{1} \lambda_{1}\left(v_{1}\left(r_{2} \lambda_{2}\right) \bar{H}_{2}\left(r_{2} \lambda_{2}\right)+\bar{V}_{1}\left(r_{2} \lambda_{2}\right)\right)\right) \\
& +\left(r_{1} \lambda_{1}+r_{2} \lambda_{2}\right)\left(1+r_{2} \lambda_{2} \bar{H}_{1}\left(r_{1} \lambda_{1}\right)+r_{1} \lambda_{1} \bar{H}_{2}\left(r_{2} \lambda_{2}\right)\right) \\
& \left.\overline{V_{2}}\left(r_{1} \lambda_{1}+r_{2} \lambda_{2}\right)\right) /\left(-r_{2} \lambda_{2}+r_{1} \lambda_{1}\left(-1+\left(v_{1}\left(r_{2} \lambda_{2}\right)\right.\right.\right. \\
& \left.v_{2}\left(r_{1} \lambda_{1}+r_{2} \lambda_{2}\right)+\left(r_{1} \lambda_{1}+r_{2} \lambda_{2}\right) \bar{V}_{2}\left(r_{1} \lambda_{1}+r_{2} \lambda_{2}\right)\right) \\
& \left.h_{1}^{*}\left(r_{2} \lambda_{2}\right)\right)+r_{2} \lambda_{2}\left(v_{1}\left(r_{1} \lambda_{1}\right) v_{2}\left(r_{1} \lambda_{1}+r_{2} \lambda_{2}\right)+\left(r_{1} \lambda_{1}\right.\right. \\
& \left.\left.\left.+r_{2} \lambda_{2}\right) \overline{V_{2}}\left(r_{1} \lambda_{1}+r_{2} \lambda_{2}\right)\right) h_{2}{ }^{*}\left(r_{1} \lambda_{1}\right)\right)
\end{aligned}
$$

\section{REFERENCES}

[1] Li, Z. and Zhao, P. 2007. Reliability analysis on theshock model of complex systems, IEEE Transactions on Reliability 56 (2) 340-348.

[2] El-Sherbeny. S. M. 2013. Stochastic analysis of a two non-identical unit parallel system with different types of failures subject to preventive maintenance and repairs. Hindawi Publishing Corporation Mathematical Problems in Engineering (Volume 2013).

[3] El-Sherbeny. S. M. 2012. Cost benefit analysis of series systems with mixed standby components and $k$-stage Erlang repair time. International Journal of Probability and Statistics.1 (2) 11-18.

[4] Mahmoud, M. A. W. and Moshref, M. E. 2010. On a two-unit cold standby system considering hardware, human error failures and preventive maintenance, Mathematical and Computer Modelling 51 (5-6)736745 .

[5] El-Sherbeny. S. M. 2010. The optimal system for series systems with mixed standby components. Journal of Quality in Maintenance Engineering 16 (3) 319-334.

[6] El-Sherbeny. S. M. 2012. Optimal system for warm standby components in the presence of standby switching failures, two types of failures and general repair time.International Journal of Computer Applications 44 (20)

[7] Doshi, B. T. 1986. Queueing systems with vacations - A survey, Queueing Systems 1 (1) 29-66.

[8] Ke. J. C. and Wang. K. H. 2007. Vacation policies for machine repair problem with two type spares, Applied Mathematical Modeling 31 (5) 880-894.

[9] Goel, L. R. and Shrivastava, P. 1992. Comparison of reliability characteristics of two (double component) systems with bivariate exponential lifetimes, Int. J. Systems Sci, Vol.23, No.1, 135-144. 
[10] Jia, J. and Wu, S. 2009. A replacement policy for a repairable system with its repairman having multiple vacations, Computers and Industrial Engineering 57 (1) $156-160$

[11] Yuan, L. and Xu, J. 2011. A deteriorating system with its repairman having multiple vacations, Applied Mathematics and Computation 217 (10) 4980-4989.
[12] Wu, Q. and Wu, S. 2011. Reliability analysis of two-unit cold standby repairable systems under Poisson shocks, Applied Mathematics and computation 218 171-182.

[13] Chen, Y., Meng, X. and Chen, S. 2014. Reliability analysis of a cold standby system with imperfect repair and under poisson shocks, Hindawi Publishing Corporation Mathematical Problems in Engineering, (Volume 2014), Article ID 507846, 11 pages. 